\title{
PI3K/BET Inhibitor LY294002
}

National Cancer Institute

\section{Source}

National Cancer Institute. PI3K/BET Inhibitor LY294002. NCI Thesaurus. Code C148229.

A morpholine-based inhibitor of phosphatidylinositol 3-kinase (PI3K) and the bromodomain and extra-terminal (BET) family of proteins, with potential antineoplastic activity. Upon administration, the PI3K/BET inhibitor LY294002 specifically targets and binds to both PI3K and the acetylated lysine recog nition motifs in the bromodomains of BET proteins. Inhibition of PI3K activity inhibits the PI3K/AKT kinase signaling pathway. This may result in inhibition of growth and survival for tumor cells in which the PI3Kmediated signaling pathway is overactivated. Inhibition of BET proteins prevents their interaction with acetylated histones, disrupts chromatin remodeling and inhibits the expression of oncogenic drivers that are important for cell proliferation and survival, which together may lead to an inhibition of proliferation in BET-overexpressing tumor cells. Activation of the PI3K signaling pathway is frequently associated with tumorigenesis. BET proteins, comprised of BRD2, BRD3, BRD4 and BRDT, are transcriptional regulators and play an important role during development and cellular growth. In tumor cells, BET proteins play a key role in the regulation of oncogene transcription and tumor cell proliferation. 